Estudios sobre armas antiguas, arte militar $\mathrm{y}$ vida cultural en oriente y occidente

XXXIX (2019), pp. 109-126

ISSN: 0436-029X

https://doi.org/10.3989/gladius.2019.06

\title{
UNE DAGUE À OREILLES DU XVe SIECLE ET SON FOURREAU DÉCOUVERTS À CASTEL-MINIER (PYRÉNÉES FRANÇAISES)
}

\author{
A 15TH CENTURY EAR-DAGGER AND ITS SCABBARD FROM THE FOUND \\ AT CASTEL-MINIER (FRENCH PYRENEES)
}

POR

Florian Téreygeol ${ }^{*}$, Alexandre Disser ${ }^{*}$, Julien Flament* et Guillaume Sarah ${ }^{* *}$

\section{ReSUME - ABstract}

Il est peu commun de mettre au jour de l'armement dans un contexte archéologique minier. La découverte faite à Castel-Minier est d'autant plus importante qu'elle est intervenue dans un contexte archéologique maitrisé. Elle met en lumière une dague à oreilles de la fin du XV siècle qui diffère largement de celles connues dans les collections par sa sobriété. C'est la première fois que cet équipement offensif est découvert en contexte archéologique. $\mathrm{Au}$-delà de la raison d'être de ce poignard dans une mine d'argent au XVe siècle, son étude permet de mettre en lumière pour la première fois une dague à oreilles de facture catalane destinée à un marché bien différent de celui des exemplaires connus dans la bibliographie.

Unearthing a weapon in an ancient mining area during an excavation is very unlikely. The discovery made at Castel-Minier is even more important since its stratigraphical context is well characterized, dating from the $15^{\text {th }}$ century. This ear-dagger of an uncommon type, found along with its scabbard, differs greatly from those known in the museum collections by its sobriety. An archaeometric study was carried out focusing on the chemical characterization of its distinct components, putting in light the choice of the assembled materials (wood, gold, lead and copper alloys). Beyond the purpose of this dagger lost in a $15^{\text {th }}$ century silver mine, its study makes it possible to see for the first time a Catalan area ear dagger produced for a regional market.

\section{Mots-CLES - KEYWORDS}

Dague à oreilles; Castel-Minier; alliages cuivreux; typologie; $\mathrm{XV}^{\mathrm{e}}$ siècle.

Ear dagger; Castel-Minier; copper alloys; typology; $15^{\text {th }}$ century.

\section{COMment Citer CET ARTicle / CitATion}

Téreygeol, F., Disser, A., Flament, F. y Sarah, G. (2019): «Une dague à oreilles du XVe siecle et son fourreau découverts à Castel-Minier (Pyrénées Françaises)». Gladius, XXXIX: 109-126. https://doi.org/10.3989/gladius. 2019.06

* UMR 5060 IRAMAT-LMC, LAPA, NIMBE, CEA, CNRS, Université Paris-Saclay, CEA Saclay 91191 Gif-surYvette France, tereygeol@cea.fr / ORCID iD: https://orcid.org/0000-0003-0629-2473; https://orcid.org/0000-0003-09142606

** UMR 5060 IRAMAT-Centre Ernest Babelon, CNRS, Université d'Orléans / https://orcid.org/0000-0003-42115001 ; https://orcid.org/0000-0002-3907-2074 


\section{INTRODUCTION}

Au cours du $\mathrm{XX}^{\mathrm{e}}$ siècle, plusieurs auteurs se sont intéressés à ce qu'il est convenu d'appeler les dagues à oreilles (ear dagger, alla Levantina, ou encore stradiote). Il s'agit d'un poignard d'inspiration arabe qui serait apparu en Europe dès le XIII ${ }^{\mathrm{e}}$ siècle, et qui fait l'objet d'un effet de mode entre la fin du XVe siècle et le XVI ${ }^{\mathrm{e}}$ siècle. En 1964, Rodríguez Lorente en a proposé une typologie critique selon trois types distincts, recentrant l'origine de cette arme en Espagne mauresque (Rodríguez Lorente, 1964). Suite à la découverte d'une dague de ce type dans un contexte archéologique daté et documenté précisément, et grâce aux études archéométriques qui ont été menées par la suite, il nous a semblé pertinent de mettre cet objet en perspective d'un corpus actualisé. Il s'agit d'insister sur les caractéristiques qui font de notre objet un unicum parmi les pièces de la même famille.

\section{CONTEXTE ET DATATION DE LA DECOUVERTE}

Le site de Castel-Minier dans les Pyrénées centrales françaises (département de l’Ariège) constitue un ensemble archéologique de premier plan pour l'étude des mines et de la métallurgie à la période médiévale. Positionnée sur un gisement polymétallique d'argent, de cuivre et de plomb, l'activité métallurgique s'y est déroulée de la fin du XIII ${ }^{\mathrm{e}}$ siècle jusqu'autour des années 1580. Dès le XIV ${ }^{\mathrm{e}}$ siècle, il s'y développe conjointement une production de fer grâce à des importations de minerai d'une vallée voisine. Fait suffisamment rare pour être mentionné, l'exploitation de cette mine et de ces ateliers est connue au travers de plusieurs sources d'archives. Dans la première moitié du XIV siècle, un conflit dont l'issue ne nous est pas parvenue oppose les exploitants du lieu, le vicomte de Couserans puis les officiers royaux, au cours d'un long procès (Verna, 1996). En 1340, le florentin Girardi, maître des mines du roi de Navarre, rapporte les multiples étapes de ce procès (Mugueta, 2004). Il invite son souverain à ne pas suivre l'exemple des mines d'Aulus (bail emphytéotique donné aux exploitants) et à privilégier une exploitation en propre des ressources minières de son royaume. Enfin, moins

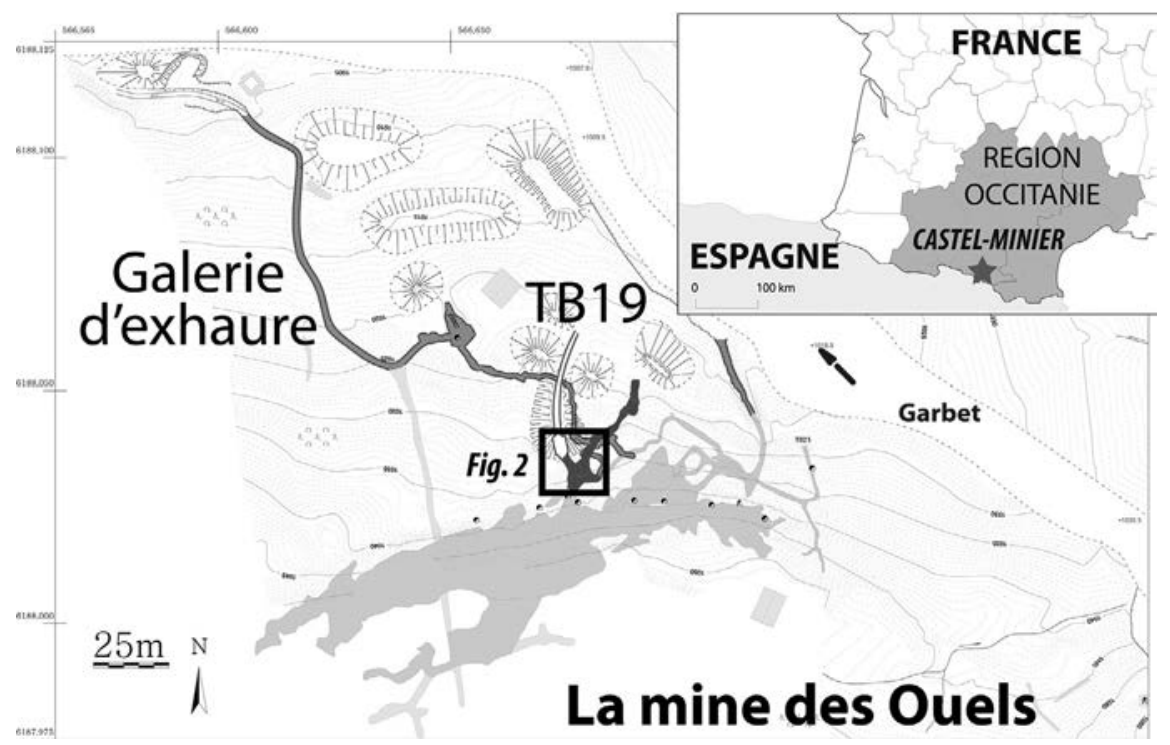

Figura 1. Localisation du site et zone de découverte de la dague. 
de 20 ans après la cessation de l'activité en ce lieu, Jean de Malus, maître de la Monnaie de Bordeaux agissant pour le compte du Roi de France, donne le récit de sa visite du site et du parcours qu'il a réalisé dans le réseau minier (Malus, 1600 : 70-84). Le site de Castel-Minier s'organise en deux pôles que sépare un torrent : en rive gauche, l'ensemble minier des Ouels, en rive droite, le castrum et les ateliers métallurgiques.

Les fouilles conduites à Castel-Minier depuis 2003 ont permis d'affiner la chronologie de l'exploitation et ont livré un riche corpus de structures et de mobilier en lien avec les métallurgies du fer et des métaux non-ferreux (Téreygeol, 2016). Parallèlement, du mobilier en relation avec la vie domestique qui s'organise autour du castrum a été mis au jour. Ce castrum est édifié vers la fin du XIV siècle et demeure actif jusqu'à la fin du XV siècle. Au regard du procès, sa mise en place semblerait marquer la reprise en main de l'exploitation par le seigneur local, le vicomte de Couserans. Cet ensemble castral se limite à une tour et une enceinte couvrant $800 \mathrm{~m}^{2}$. Marque de l'autorité plus que lieu d'habitat, il a pu accueillir quelques agents du seigneur en charge du contrôle de la production du métal précieux.

La mine se développe directement en regard du castrum. Exploitée dès la fin du XIII ${ }^{\mathrm{e}}$ siècle, elle connait au $\mathrm{XV}^{\mathrm{e}}$ siècle des investissements importants, mais vains, pour atteindre des niveaux profonds : l'installation d'un treuil puissant en entrée de mine, l'aménagement d'une galerie supérieure pour l'évacuation des eaux, et le percement sur $120 \mathrm{~m}$ de long d'une galerie d'exhaure restée inachevée.

La dague que nous présentons a été découverte à l'occasion de la fouille d'une des entrées de cette mine. Elle a été mise au jour au fond d'une fosse creusée dans la roche et barrant l'entrée. Elle reposait sous deux bois de mine. Son état de conservation est exceptionnel pour ce qui concerne les parties en bois et en métaux non ferreux. En revanche les éléments en fer ont dû jouer le rôle de cathode sacrificielle et se trouvent particulièrement altérés. Il en va de

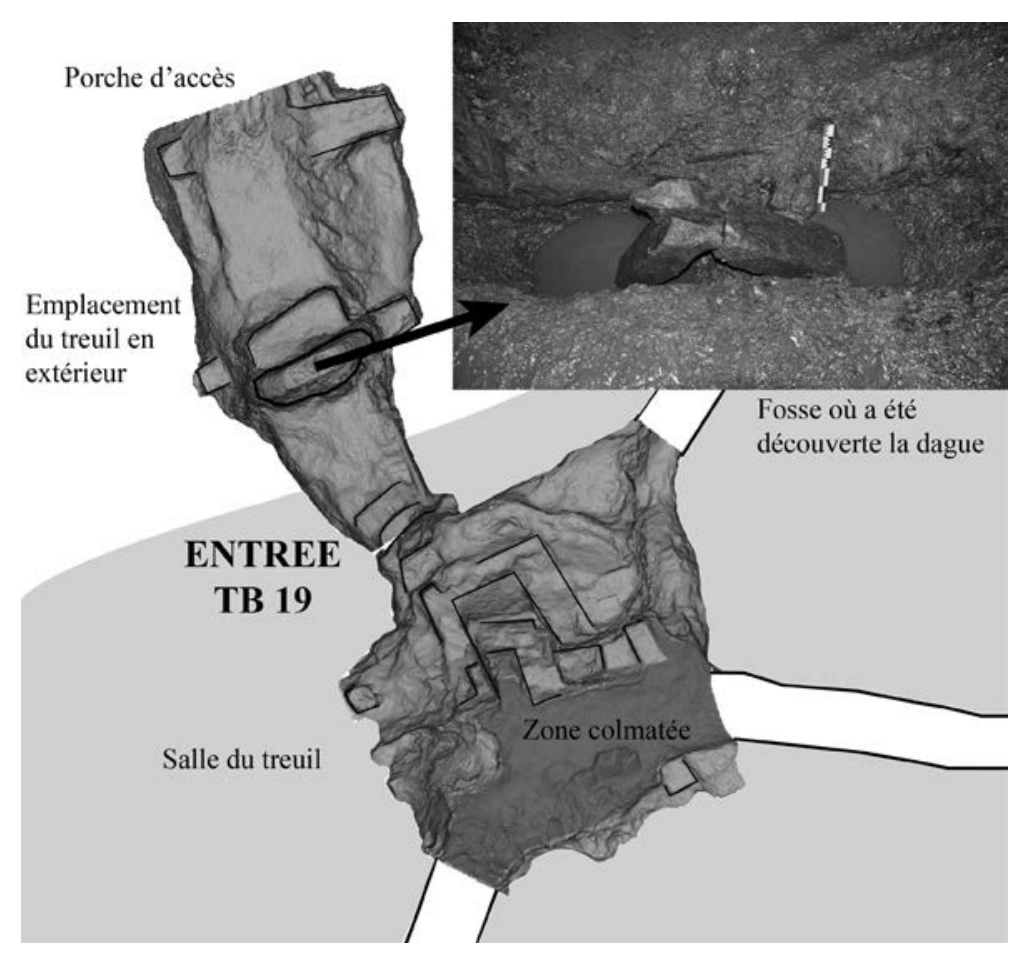

Figura 2. Plan de la zone et photo de la fosse. 
même pour le fourreau dont seules quelques traces subsistent. La datation de l'objet est faite à partir de son contexte de découverte et de la séquence d'exploitation de la mine que nous avons pu restituer par ailleurs. Tout d'abord, une étude dendrochronologique a révélé que les bois de mine retrouvés dans le niveau supérieur du comblement ont été coupés en 1397-1398. Cette date marque évidemment le terminus post quem puisqu'elle reflète le début de l'activité dans cet espace. La dague n'a donc pu être perdue qu'après cette date malgré le positionnement stratigraphique. Le lieu de découverte correspond à un réaménagement qui s'inscrit dans un ensemble plus vaste lui aussi bien daté grâce à la découverte d'une monnaie. Il s'agit d'un liard au dauphin de Louis IX frappé entre 1467 et 1483 en Normandie. Si l'on envisage un délai de quelques années entre l'origine de cette pièce dans le nord de la France et sa perte dans les Pyrénées, alors l'entrée de cette mine devait encore être fréquentée dans le dernier quart du $\mathrm{XV}^{\mathrm{e}}$ siècle. Enfin, la galerie d'exhaure avortée qui devait venir remplacer le treuil à l'emplacement duquel nous avons trouvé la dague a livré un corpus d'outils de mineur. Un de ces outils a été daté par radiocarbone. Sa perte ou son abandon remonte entre 1445 et 1515 . Ainsi la dague aurait été perdue entre 1483 et 1515 . Cette fourchette chronologique s'accorde parfaitement avec les datations de dagues à oreilles connues dans la littérature. Il faut déjà noter à ce stade qu'il s'agit de l'unique dague à oreilles dont la découverte ait été faite en contexte de fouille archéologique.

\section{LA DAGUE A OREILLES DE CASTEL-MINIER}

Après son dessin et la couverture photographique, une série de radiographies a été faite avant la restauration. Dans le même temps, nous avons procédé à des analyses par fluorescence des rayons $\mathrm{X}$ (XRF) sur les parties non oxydées des différents éléments métalliques qui

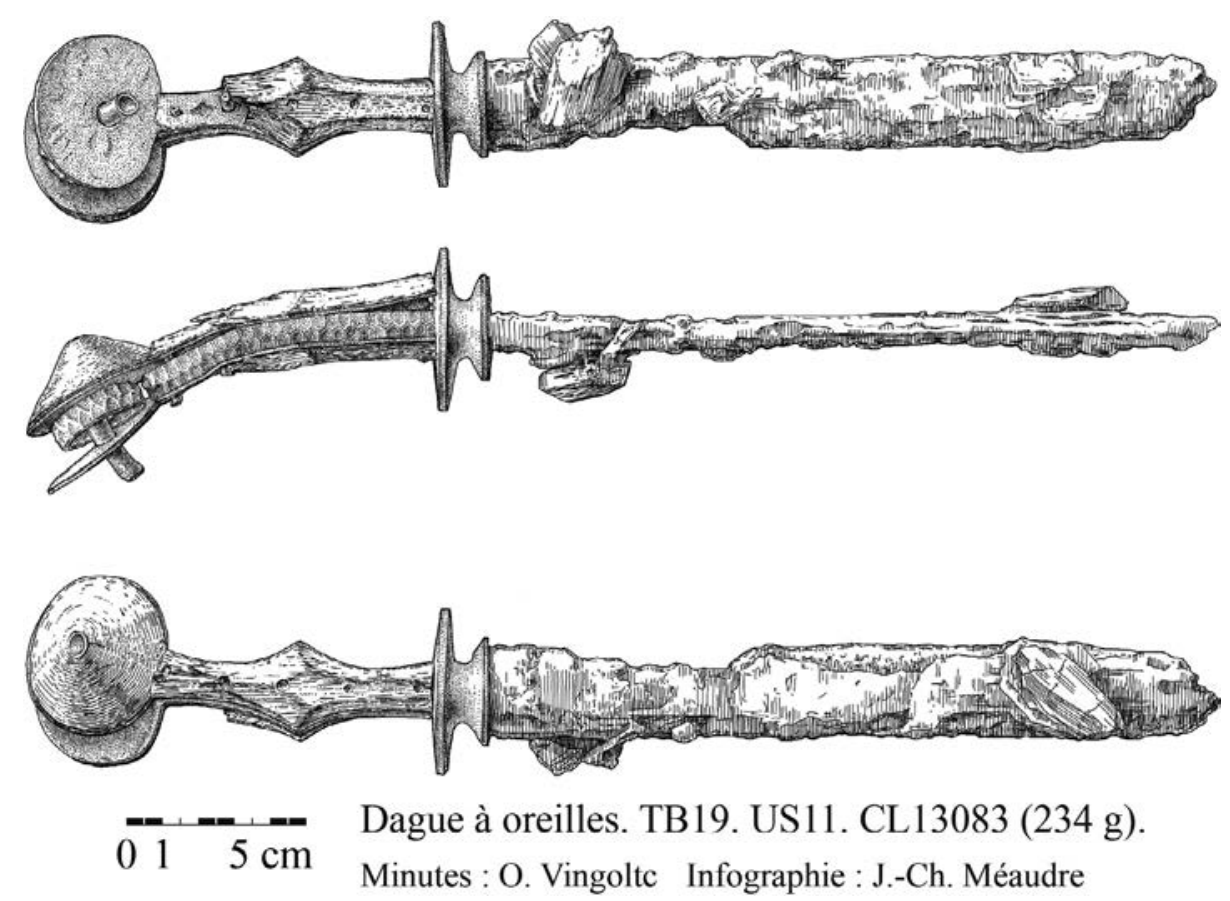

Figura 3. Dessins de la dague avant restauration. 
composent cette arme. L'objet, d'une longueur de 33,4 cm pour un largueur aux oreilles de 4,5 $\mathrm{cm}$, a été restauré et stabilisé ultérieurement ce qui a apporté un nouveau lot d'informations et rendu possible de nouvelles analyses.

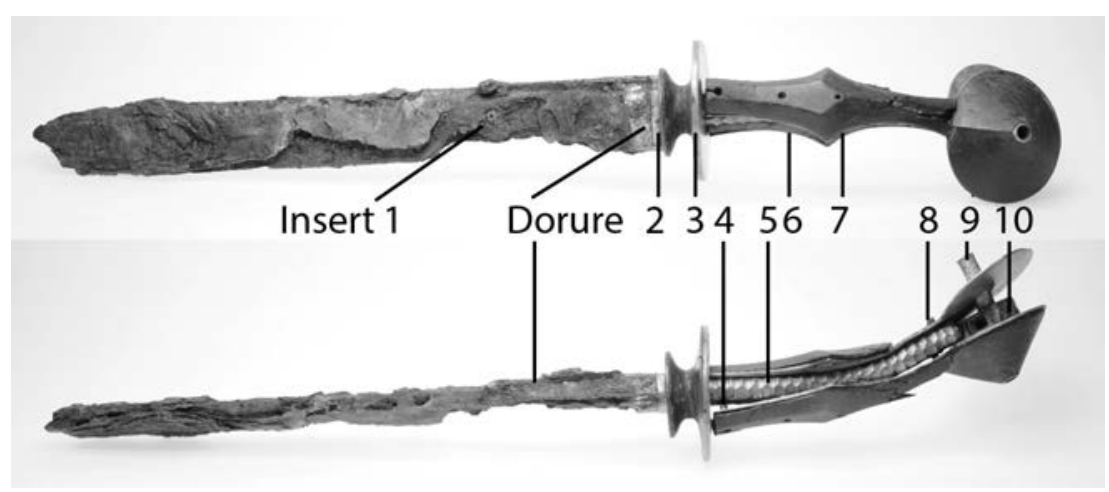

Figura 4. Photographie après restauration avec localisation des analyses.

\section{LE FOURREAU}

L'objet se compose de la dague proprement dite et des vestiges de son fourreau. Celui-ci n'a été détecté que tardivement, après la restauration. Nous n'en conservons que deux témoignages : la chape et le négatif d'un décor. Sur le corps de la lame, la concrétion ferrugineuse a soudé un fragment de schiste. Après retrait de cette pierre, nous avons pu identifier sans doute possible le négatif d'un décor en treillis appliqué probablement par gaufrage sur cuir. Les fourreaux ainsi décorés ont été trouvés assez fréquemment (Cowgill et alii, 1987).

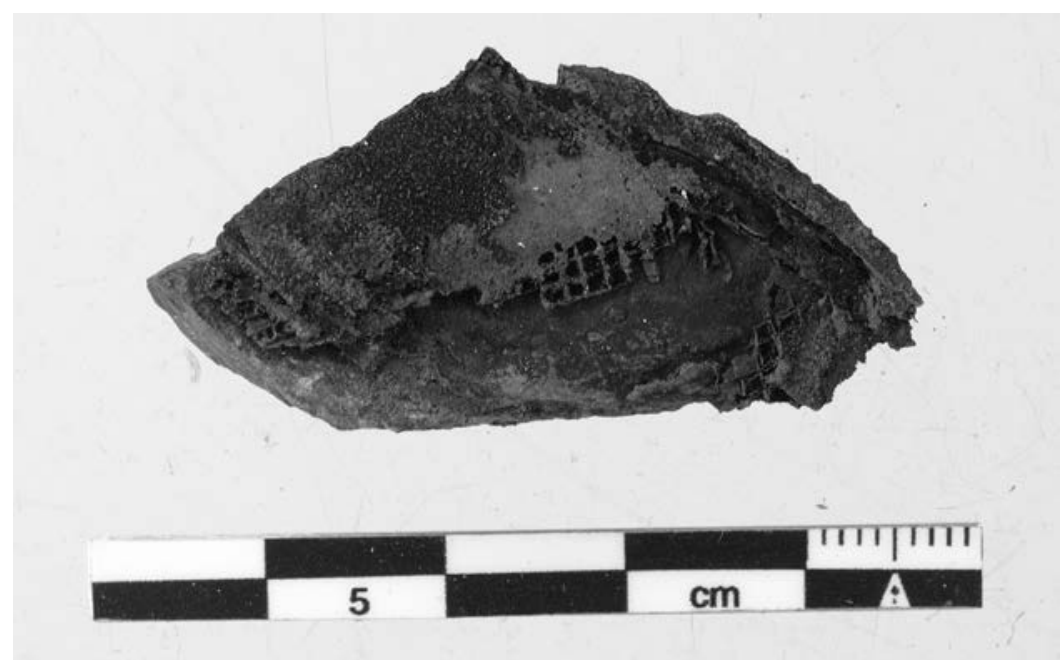

Figura 5. Photographie du négatif du décor de cuir.

La chape se composait vraisemblablement d'une tôle en laiton dont le contact avec la garde est ourlé, formant une bande de $4 \mathrm{~mm}$ de large. Dans sa partie inférieure, une dorure dessine un bandeau d'or en biseau d'une largeur 0,6 à $1 \mathrm{~cm}$. Des fragments de dorure sont 
encore visibles sur le corps du fourreau, mais sans qu'une approche stylistique soit possible. Grâce à la radiographie, nous avons mis en évidence sur les tranches du fourreau un dépôt d'or sur une longueur de 5,2 cm d'un côté et de 0,6 cm de l'autre. Ces dépôts sont au contact de la dorure ourlant la chape. Il manque probablement à cet ensemble une bouterolle venant terminer le fourreau.

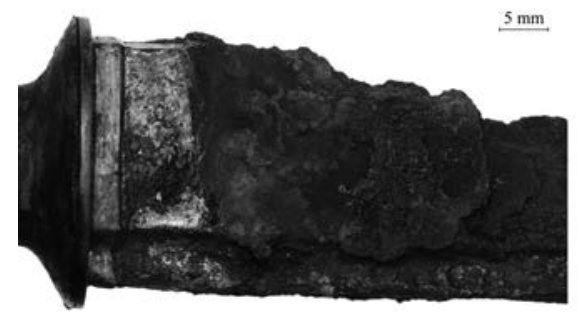

Figura 6. Photographie de la chape du fourreau.

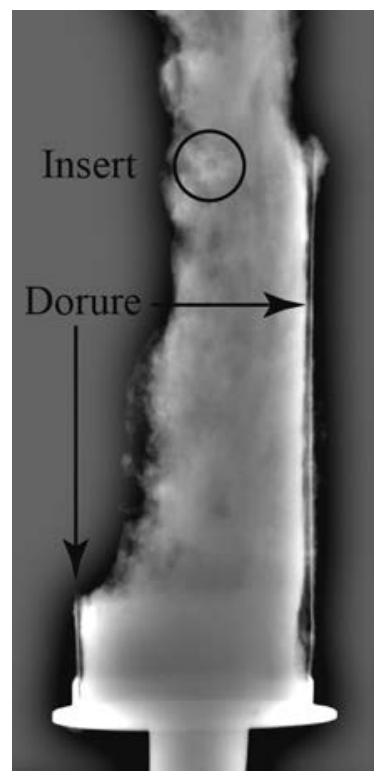

Figura 7. Détail de la radiographie montrant l'or se développant sur la tranche et la marque de fabrique.

\section{LA LAME DE LA DAGUE}

La lame est la partie de l'objet qui a le plus souffert de son enfouissement en milieu humide. De plus, étant conservée dans son fourreau, elle est particulièrement difficile à décrire, mais ses dimensions avoisinent les moyennes du corpus connu. Sa longueur conservée est de $20 \mathrm{~cm}$. Nous ne savons pas si elle est complète. Sa largeur est estimée à $1,9 \mathrm{~cm}$ pour une épaisseur de $0,3 \mathrm{~cm}$. Cette dernière mesure est sujette à caution du fait de l'état de conservation. Elle s'accorde cependant avec ce qui a pu être observé sur des lames bien préservées.

Seuls deux indices permettraient d'affirmer la présence d'un ricasso dissymétrique assez prononcé. Il s'agit pour le premier de la forme même du fourreau dont ses tranches montrant 
deux plats dorés longs pour l'un de 5,6 $\mathrm{cm}$ et de $1 \mathrm{~cm}$ pour l'autre. Le second indice est le dépôt d'or en biseau qui orne la chape et évoque cette dissymétrie du ricasso. Selon Rodriguez Lorente, la forme et la dimension de cette partie de la lame sont des caractéristiques typologiques importantes. Les modèles arabes présentent des ricassi dissymétriques très développés le long de la lame. Au travers du corpus existant, les pièces sans ricasso sont au nombre de cinq et jamais d'origine espagnole. Le ricasso symétrique est caractéristique du type II (à l'exception d'un des exemplaires de la bibliothèque Ambrosiana). Le type III comporte certes une majorité de ricassi dissymétriques (quinze exemplaires contre dix exemplaires symétriques), mais le corpus est trop faible pour dégager une tendance significative.

Sur la lame, la radiographie a révélé la présence d'une marque de fabrication damasquinée: une rouelle à six crans. La restauration a permis de la révéler parfaitement. Cette petite pièce ajoutée est centrée sur l'axe de la lame. Sur les autres dagues, lorsqu'un poinçon existe avec ou sans insert, il se trouve toujours décalé vers l'un des tranchants. Pour les dix cas inventoriés, cinq présentent une gravure simple, les autres sont des damasquinures de cuivre, ou d'or dans un cas. L'usage d'un poinçon n'est pas rare en coutellerie et il serait vain de chercher une corrélation avec d'autres pièces, d'autant plus que le coutelier qui a forgé cette lame n'est pas nécessairement le fourbisseur qui a assemblé la dague.

\section{LA POIGNÉE}

La garde est composée de deux troncs de cônes de bois réunis sur leur plus petite base de façon à former une poulie. Deux rondelles de laiton viennent supporter les grandes bases des cônes. Celle au contact du manche est dotée d'un repli qui vient enserrer la base de la poulie et assurer son maintien. Celle au contact de la lame est plate.

Deux platines en laiton préfigurent la forme finale de la poignée. Elles reçoivent les plaquettes en bois. L'ensemble est solidarisé par cinq rivets faits de feuilles de laiton roulées traversant toutes les pièces, soie comprise. La radiographie a montré l'existence d'un vide élargi à la base de chaque percement dans le bois, qui s'apparente à une série d'avant-trous de centrage disposés de façon à être invisibles. Un fil de métal a également été détecté dans le bois sans que sa présence puisse trouver une justification. Sur la tranche, la soie est couverte par l'apport de deux lamelles de laiton épaisses $(8,3 \mathrm{~cm}$ de long, $0,7 \mathrm{~cm}$ de large et $0,5 \mathrm{~mm}$ d'épaisseur) avec un décor guilloché. Ces lamelles sont soudées directement sur les platines.

L'essence de bois utilisée n'a pu être déterminée qu'à partir des plaquettes formant la poignée. Il s'agit d'un bois de la famille des Rosacea. Le genre est soit Pyrus soit Sorbus. Étant donnée la fonction de l'objet, il est probable qu'il s'agisse d'un bois de poirier. L'identification ne peut être plus poussée sans risque pour l'intégrité de l'objet. Le choix de ce bois serait cohérent avec l'usage prévu pour cet objet puisque le poirier offre une matière dense, un grain fin et une bonne résistance. Neuf, le manche devait avoir une teinte rosâtre qui s'équilibre bien avec la couleur du laiton qui le porte.

Les platines de laiton formant le support des plaquettes de bois se développent pour donner la forme du pommeau caractéristique de la dague à oreilles. Une seule des oreilles est complète. Elle est formée d'un cône de bois ayant $1,7 \mathrm{~cm}$ de hauteur. La fixation de l'ensemble est assurée par un jeu de deux rivets de laiton, chacun fait d'une feuille roulée sur elle-même. Les extrémités cachées ont été taillées de façon à venir se bloquer sur la soie. Les oreilles sont disposées dans l'axe du manche et ne présentent pas l'évasement si caractéristique dans le corpus connu. Selon plusieurs auteurs, cet écartement des oreilles permet de saisir le poignard en positionnant son pouce entre les deux oreilles, en appui dans l'axe de la lame. Le coup porté 
serait plus fort et l'homme plus difficile à désarmer. Dans notre cas, ce mode de préhension n'est pas possible et la dague doit être tenue en refermant le pouce sur les doigts.

Un anneau terminal avec un méplat rend invisible le système de fixation des oreilles. L'anneau est doté de deux percements carrés dans lesquels la terminaison de la soie s'emboitait. Comme pour les tranches rapportées du manche, un guillochage assure sa décoration.

\section{APPROCHE ANALYTIQUE}

L'approche analytique a été conduite en deux temps. D'abord, les métaux non oxydés ont été analysés immédiatement après la découverte. Ce n'est qu'après la restauration que nous avons pu travailler sur la chape, l'insert de la lame et sur la soudure des dos. Parmi les moyens à notre disposition, seul l'usage de générateurs de rayons $\mathrm{X}$ était pertinent puisqu'il permettait de respecter l'intégrité de l'objet. Les analyses des alliages cuivreux ont été conduites à l'IRAMAT-Centre Ernest Babelon. Les calibrations propres à l'appareil, établies grâce à des matériaux de référence aux teneurs certifiées, nous autorisent à proposer des résultats quantitatifs. La dorure et la soudure ont été observées à l'aide de la fluorescence des rayons X par anode tournante (au molybdène) au sein du LAPA. Dans ces derniers cas, il s'agit uniquement d'analyses qualitatives.

\section{LA NATURE DES ALLIAGES CUIVREUX}

Les alliages cuivreux sont mis en œuvre à différents endroits de l'objet : les rondelles de la garde, les platines de la poignée, les plaques de décoration, l'insert sur la lame et les rivets du manche et des oreilles. Parmi la gamme des alliages cuivreux disponibles réunis dans le diagramme ci-dessous, il y a un usage exclusif des laitons. En fonction de la destination de la pièce et surtout de la visibilité sur l'objet fini, il y a un choix réfléchi des natures d'alliages.

En reprenant la nomenclature établie par J. Bayley, que nous actualisons, il ne se distingue pas moins de trois gammes d'alliages mis en œuvre pour réaliser cette dague : le laiton, le laiton rouge et le laiton au plomb. Le laiton est utilisé pour les pièces les plus visibles : la garde,

Figura 8. Tableau des résultats analytiques, opérateurs : J. Flament, G. Sarah, IRAMAT-CEB, Orléans (voir la figure 4 pour la localisation des analyses).

\begin{tabular}{|c|c|c|c|c|c|c|c|c|c|}
\hline N $^{\circ}$ D'ANALYSE & PièCE & Cu (\%) & $Z_{\mathrm{N}}(\%)$ & $\mathrm{SN}_{\mathrm{N}}(\%)$ & Pв (\%) & $\mathrm{FE}_{(\%)}$ & $\mathrm{N}_{\text {I }}(\%)$ & $\mathrm{SB}_{\text {( \%) }}$ & TотAL \\
\hline 1 & Insert & 83,9 & 7,2 & 7,9 & 1,0 & - & - & - & 100 \\
\hline 2 & Chappe & 87,0 & 7,2 & 4,7 & 1,1 & - & 0,05 & - & 100 \\
\hline 3 & Garde & 71,8 & 26,9 & 0,06 & 0,56 & 0,22 & 0,24 & 0,19 & 100 \\
\hline 4 & Rivet & 78,3 & 12,8 & 0,11 & 7,6 & 0,30 & 0,78 & 0,13 & 100 \\
\hline 5 & Guillochage & 69,0 & 26,6 & 1,1 & 1,4 & - & 0,28 & 1,7 & 100 \\
\hline 6 & Platine & 85,7 & 7,1 & 5,54 & 0,84 & 0,26 & 0,05 & 0,51 & 100 \\
\hline 7 & Platine & 83,4 & 7,4 & 6,1 & 1,2 & 1,9 & 0,05 & - & 100 \\
\hline 8 & Rivet & 79,4 & 4,3 & 2,7 & 13,3 & - & 0,25 & - & 100 \\
\hline 9 & Rivet & 85,4 & 7,2 & 5,48 & 0,93 & 0,43 & 0,05 & 0,55 & 100 \\
\hline 10 & Anneau & 70,6 & 27,4 & 0,55 & 1,2 & - & 0,27 & - & 100 \\
\hline
\end{tabular}


l'anneau et les dos guillochés. La teneur en zinc est égale ou supérieure à $24 \%$, conférant à chaque pièce un bel aspect doré. Les discrets rivets du manche sont quant à eux en laiton au plomb, alliage plus terne. Le laiton rouge, alliage ternaire cuivre-zinc-étain rappelant la couleur du bois de poirier, est préféré pour les platines et le rivet des oreilles. Il forme également l'ourlet de la chape. Enfin l'insert en étoile dans la lame est aussi dans la gamme des laitons rouges, mais d'une qualité supérieure, puisque sans trace de plomb.

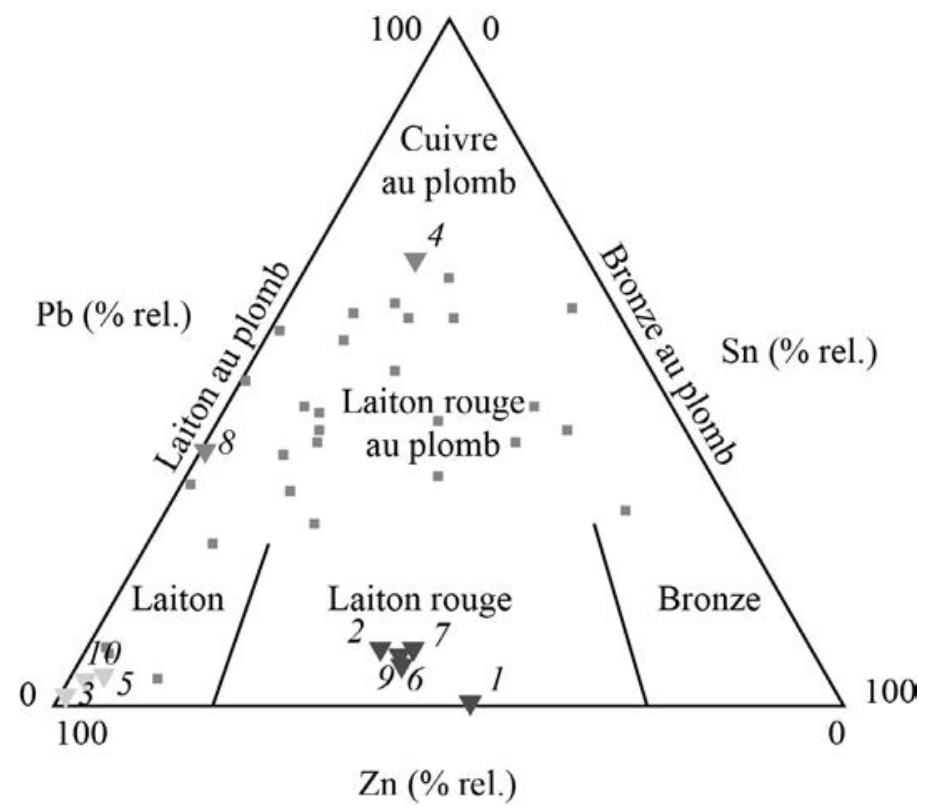

Figura 9. Distribution sur le diagramme ternaire des natures d'alliages employées (les triangles numérotés renvoient aux analyses, voir Figura 8 et 4 ; les carrés représentent les analyses des pommeaux faites par Dandridge et Wypyski en 2011).

Il y a ici une finalité esthétique évidente. Le soin apporté à la réalisation de cette dague devient encore plus flagrant si l'on compare ces choix d'alliages à un des seuls corpus d'objets de coutellerie analysés : les pommeaux provenant de dagues et d'épées des Croisades (Dandridge et Wypyski, 2011). Le nombre d'objets analysés s'élève à vingt-huit et définit un usage préférentiel des laitons rouges au plomb. Seuls trois de ces pommeaux sont en laiton avec une teneur en zinc n'excédant pas $20 \%$ alors qu'il s'agit des zones les plus visibles de la poignée. Le laiton rouge au plomb $(\mathrm{Cu}-\mathrm{Zn}-\mathrm{Sn}-\mathrm{Pb})$ doit être considéré comme un assemblage de faible valeur relative issu du recyclage par fonte de différents objets. À l'inverse le laiton est une réalisation volontaire par cémentation de cuivre avec du minerai de zinc dont la couleur dorée dépend de la teneur en zinc.

\section{LA DORURE}

La dorure qui se trouve sur la chape du fourreau vient rehausser la couleur de celle-ci. Elle se développe sur les quatre faces. Faute de calibration de l'analyse XRF, la donnée quantitative de cette dorure reste inaccessible, en revanche le spectre de fluorescence permet de caractériser le mode de dépôt. La présence de mercure montre à l'évidence qu'il s'agit d'une dorure à l'amalgame. Ce dernier est étalé sur la zone à dorer, puis il est chauffé pour évaporer le 
mercure. La finition par polissage ou brunissage révèle la couleur de l'or et donne une couche d'une épaisseur autour de 13 microns.

\section{LA BRASURE}

Enfin, les contacts entre les platines et les dos guillochés montrent un assemblage par la technique de la brasure (Arminjon, 1998: 322). L'une, sûrement la première réalisée, est correctement exécutée. Elle se développe sur toute la longueur du dos $(7,4 \mathrm{~cm})$. Le surplus de matière est difficile à déceler. Réalisée dans le cours du montage du manche, l'autre brasure montre des épatements venant empiéter autant sur les platines que sur les dos. Elle est localisée de part et d'autre du rivet central et mesure seulement $3,8 \mathrm{~cm}$ de long. Il est difficile de dire si la brasure permet au dos d'adhérer à la soie, en plus de la platine. La radiographie n'apporte pas de réponse. Une double analyse par diffraction des rayons X puis par dosage des électrons de diffusion secondaire montre que les éléments composant cette soudure sont du soufre et du fer sous la forme d'une pyrite. Il s'agit d'une substitution post-dépositionnelle évidente de la matière originelle. Les conditions environnementales de la découverte s'y prêtent : une fosse humide avec de la matière organique et la présence géologique d'éléments sulfurés. Reste ainsi la question de la composition originelle de la brasure. Les possibilités d'assemblage à chaud entre deux plaques de laiton sont peu nombreuses : le plomb, l'étain ou un alliage des deux métaux. Pour braser les pièces en alliage de cuivre sur la soie en fer, la solution résiderait dans l'usage d'une brasure de bronze. Dans la mesure où les alliages de cuivre ne présentent qu'une faible altération, il est peu probable que le bronze ait été l'alliage utilisé pour braser. Nous formulons donc l'hypothèse de l'usage d'un alliage à base de plomb ne servant qu'à solidariser les dos avec les platines. Cependant, quelle que soit sa composition, il est avéré que cet assemblage était visible sur la pièce finie.

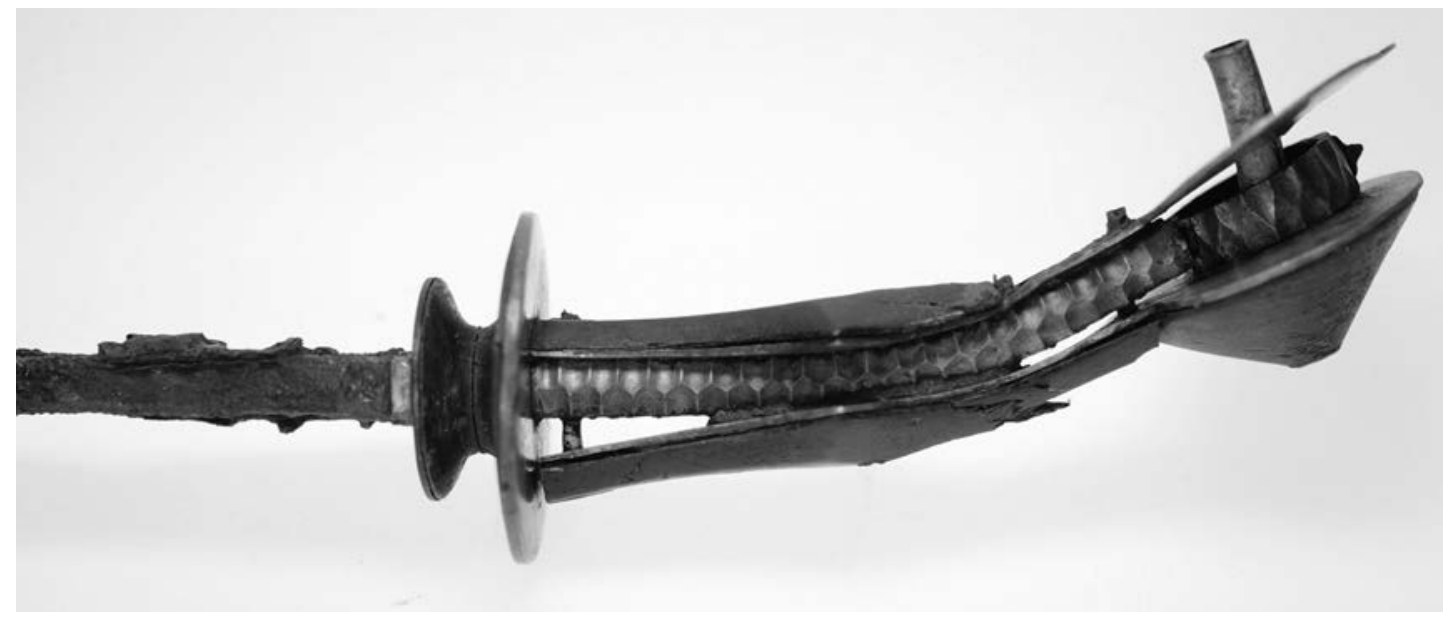

Figura 10. Détail de la soudure.

\section{COMPARAISON AVEC LA TYPOLOGIE EXISTANTE}

La dague à oreilles reste une arme assez rare. Elle est citée occasionnellement, mais fait exceptionnellement l'objet d'une description. Ainsi Oakeshott se limite à la mentionner dans 
son European weapons and armour (Oakeshott, 1980: 225) en revoyant simplement à son The archaeology of weapons (Oakeshott, 1960) dans lequel elle n'apparait cependant pas. Il faut bien reconnaitre que, comparée au cinquedea, basilard et autres dagues à rognons, la dague à oreilles n'est représentée que par un corpus réduit. Lors de sa recherche sur cet objet, Rodriguez Lorente en dénombre trente-trois en agrégeant ses propres travaux sur la péninsule ibérique, sa collection privée et les dagues déjà publiées par le Baron de Cosson (1901), Sir Laking (1920) et Bashford (1929b). Nous avons essayé à notre tour d'enrichir ce corpus à partir de la documentation disponible. Parmi les dix dagues du musée Lazaro Galdiano à Madrid (Camón Aznar, 1951), seules deux ont fait l'objet d'une publication et s'apparentent à des dagues à oreilles (Espinosa Martin, 2015: 72). Depuis 1964, il semble que le Metropolitan Museum de New York ait fait l'acquisition de trois de ces objets (https://www.metmuseum.org/: $54.46 .21 ; 32.75 .99 ; 14.25 .1259)$. En Belgique, deux exemplaires existent, l'un au Musée des Armes de Liège (référence : 10119343), l'autre au Musée Royal de l'Armée et d'Histoire Militaire de Bruxelles (référence : 11418). En France, outre l'objet de cet article, deux dagues de ce type sont connues. L'une a été publiée dans la revue du Poitou en 1918 (Charbonneau-Lassay, 1918). Il s'agit d'un exemple précoce à rapprocher de l'exemplaire trouvé à Londres (voir Laking 1920, nº 824). L'autre est conservée au musée national de la Renaissance à Ecouen sous le numéro d'inventaire ECL 11836 (https://art.rmngp.fr/fr/library/ artworks/dague-a-oreillons_acier). Enfin deux dagues ont été vendues récemment par Sotheby's et la maison Pierre Bergé et associés. Le demi-siècle qui nous sépare de la publication de Rodriguez Lorente n'a donc vu apparaitre que onze nouveaux exemplaires.

La typologie proposée par Rodríguez Lorente en 1964, au vu de la faible évolution du corpus depuis cette date, reste valide pour catégoriser les dagues à oreilles. Il distingue trois types de dagues selon des critères principalement stylistiques, mais aussi en relation avec les matériaux mis en œuvre.

Le type I est dit hispano-mauresque. Il couvre une période large (XIV-XVI ${ }^{\mathrm{e}} \mathrm{s}$.). Sa caractéristique, comme sa dénomination le laisse supposer, est d'intégrer des influences artistiques arabes. La dissymétrie du ricasso s'y trouve plus développée que sur le type III (cf. infra). Le rivet des oreilles est droit plutôt que courbe, et fait de laiton plutôt que de fer. Son emboîtement dans la soie se fait par un trou plus prismatique que cylindrique. Les oreilles et les plaquettes sont de laiton plutôt que de fer. Les oreilles d'abord en calotte évoluent vers une forme conique. Ces deux profils sont repris dans le type III italien. L'écartement entre les deux oreilles est large, facilitant la préhension avec le pouce en opposition. Cet écartement se réduira pour les types II et III. La fin du XVe siècle correspond au terminus ante quem de ce type.

Le type II, dit « dague à oreilles espagnole », a un ricasso symétrique. La poignée et la lame sont d'une même pièce forgée. Les oreilles sont atrophiées et le rivet les liant n'existe plus. La damasquinure florale ou géométrique est remplacée par des inscriptions.

Enfin le type III dit « vénitien », « italien » ou « espagnol » est une copie des dagues de type I qui apparait à la fin du $\mathrm{XV}^{\mathrm{e}}$ siècle et serait produit durant la première moitié du $\mathrm{XVI}^{\mathrm{e}}$ siècle. Certaines dagues de ce type s'accompagnent de décorations de type Renaissance. Il faut noter que les productions italiennes connaissent l'influence des stradiotes, également des dagues à oreilles, mais d'origine balkanique.

Cette classification repose, on l'a dit, sur un faible corpus. Mais elle est surtout réalisée très majoritairement à partir de dagues d'apparat à destination d'une élite, marquant un biais de conservation assez net: sur les quarante-cinq modèles que nous recensons, trente-cinq peuvent être qualifiés de luxueux. Quinze utilisent l'ivoire, dix-huit ont un ricasso ciselé, dix-neuf portent des traces de dorure sur le fer. Les apports se font par damasquinure (6), dorure (18) et niellage (2). Ces techniques sont certes répandues, mais nécessitent un véritable savoir-faire 
d'orfèvre et apportent une importante valeur ajoutée à la pièce produite. Un exemplaire présente même des ajouts de pierres et de nacre. Malheureusement les descriptions données ne permettent pas de rentrer dans les détails notamment sur les techniques de dorure et de damasquinure. S'agit-il d'incrustation de Tolède, d'azziminia, de takfit ? Nous ne sommes pas en mesure de répondre. Il en va de même pour la nature des métaux utilisés. Outre le fer, le cuivre, le laiton et le bronze semblent en usage, mais aucune analyse n'a pu être conduite. L'identification se fait à partir de la couleur. Dès lors comment détecter l'usage de bronze quand il est doré ? Comment distinguer un cuivre d'un laiton rouge, un cuivre au plomb d'un bronze au plomb ou d'un laiton rouge au plomb? Même dans le cas d'un alliage binaire comme le laiton, la variation de la teneur en zinc fait évoluer la couleur du rouge à l'or. Seule la description des pièces de la Wallace collection adopte une position prudente en mentionnant simplement l'utilisation d'un alliage cuivreux (https://wallacelive.wallacecollection.org).

Comment classer la dague à oreilles de Castel-Minier ? Par sa facture trop peu luxueuse, elle ne s'apparente à aucun des exemplaires de ce type connus. Nous pouvons bien sûr exclure l'appartenance au type II puisque poignée et lame ne sont pas d'une seule pièce. La date estimée de sa perte, entre la fin du XV $\mathrm{V}^{\mathrm{e}}$ siècle et le début du XVI $\mathrm{I}^{\mathrm{e}}$ siècle, la place au moment où ce type d'objet connait un effet de mode avec le type III. Pourtant, sa morphologie ne s'accorde pas avec les caractéristiques de ce groupe, qu'il soit italien ou espagnol. En effet, les oreilles de cette dague sont dans l'axe du manche et ne s'en écartent pas comme dans les exemplaires du corpus de référence. Pour cette même raison, la dague ne peut intégrer le groupe I, bien que l'usage du laiton et le ricasso dissymétrique la placent sous une influence arabe. La réponse ne vient pas des objets connus, mais de leur représentation. Dans l'iconographique catalane, les dagues à oreilles ont été repérées de longue date. Trois exemples sont donnés par Bashford dès 1929 (Bashford, 1929a). Rodriguez Lorente a proposé une première liste couvrant une période comprise entre 1380 et 1540 . Il montrait ainsi que les premières figurations apparaissent dès la fin du XIV ${ }^{e}$ siècle en Italie comme en Espagne, et se multiplient entre 1450 et 1550. Les représentations des dagues y sont principalement des dagues aux oreilles écartées.

Nous avons mené une recherche systématique des représentations de ces dagues par des artistes catalans de la période du gothique international dans la collection du Musée National d'Art Catalan (https://www.museunacional.cat/en/colleccio/). Une première occurrence correspondant au modèle de Castel-Minier est visible sur le retable de Sainte-Catherine et SaintEligius datant de 1400. La résurrection du Christ peinte par Guerau Gener et Lluís Borrassà en 1407-1410 montre un des gardiens du tombeau équipé de ce modèle de dague. Plus tard, entre 1442 et 1445, Bernat Martorell peint à deux reprises le martyr de Sainte-Eulalie dont le bourreau porte à chaque fois une dague à oreilles à la ceinture. Il s'agit d'ailleurs dans les deux cas du même personnage à la couleur des cheveux près. Enfin sur le bûcher de Saint-Vincent peint entre 1455 et 1460 par Jaume Huguet, le seigneur en armure porte une dague à oreilles parmi les plus luxueuses. Tous ces exemples auxquels s'ajoutent ceux déjà donnés par Rodríguez Lorente représentent des dagues dont les oreilles sont dans l'axe du manche. La relative surreprésentation picturale de ce modèle au sein de l'école catalane semble bien caractériser l'origine géographique de cet objet et indiquerait sinon une provenance, du moins une influence catalane pour la dague de Castel-Minier. Ce modèle catalan équipe des hommes d'armes assez riches pour se doter d'un matériel à la mode, mais pas suffisamment pour acquérir un modèle luxueux. Cependant si la forme de notre dague est bien en accord avec ce que nous pouvons observer sur les peintures, son fourreau reste atypique. Dans notre cas, il ne couvre que la lame. Sur les tableaux, la chape du fourreau vient enserrer la garde. Nous aurions tendance à voir une variation de style prenant place dans la seconde moitié du $\mathrm{XV}^{\mathrm{e}}$ siècle. 
章

\begin{tabular}{|c|c|c|c|c|c|c|c|c|c|c|c|c|c|c|c|c|c|c|c|c|c|c|c|}
\hline 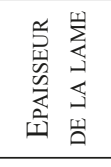 & $\begin{array}{l}\infty \\
0 \\
0\end{array}$ & $\because$ & $\begin{array}{l}\infty \\
0\end{array}$ & $\Rightarrow$ & $\hat{\sigma}$ & -7 & $\mathscr{g}$ & 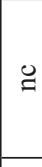 & - & $=$ & $\stackrel{2}{-}$ & $\mathscr{g}$ & $\hat{\circ}$ & $\stackrel{\mathscr{c}}{\mathscr{2}}$ & $\begin{array}{l}0 \\
0\end{array}$ & $\stackrel{U}{\Xi}$ & -1 & $\stackrel{0}{\Xi}$ & $\stackrel{0}{\Xi}$ & 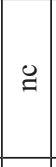 & $\mathscr{g}$ & $\stackrel{\varrho}{\Xi}$ & $\mathscr{g}$ \\
\hline 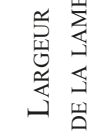 & $\approx$ & $\vec{i}$ & $\tilde{m}$ & $\vec{i}$ & $\hat{i}$ & $\begin{array}{l}n \\
i \\
i\end{array}$ & 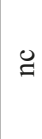 & 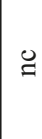 & $\begin{array}{l}\infty \\
i\end{array}$ & $\mid \begin{array}{l}n \\
i\end{array}$ & {$\left[\begin{array}{c}n \\
i\end{array}\right]$} & $\mathscr{g}$ & $\hat{i}$ & $\stackrel{\mathscr{I}}{\leftrightarrows}$ & $\approx$ & $\ddot{g}$ & $\mid \begin{array}{l}n \\
i \\
i\end{array}$ & $\stackrel{g}{g}$ & $\vec{m}$ & $\stackrel{\mathscr{a}}{\mathscr{a}}$ & $\mathscr{g}$ & $\mathscr{\Xi}$ & $v$ \\
\hline 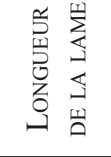 & $\vec{\sim}$ & $\mid \begin{array}{c}1 \\
\infty \\
\infty\end{array}$ & $\begin{array}{l}n \\
\tilde{v}\end{array}$ & $\begin{array}{c}0 \\
0 \\
\infty \\
-\end{array}$ & $\mid \begin{array}{l}n \\
\hat{i}\end{array}$ & $\stackrel{m}{=}$ & 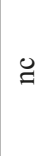 & $\mathscr{g}$ & $\hat{a}$ & $\vec{a}$ & $\overrightarrow{a j}$ & $\stackrel{\mathscr{g}}{\varrho}$ & $\stackrel{\hat{\infty}}{\infty}$ & 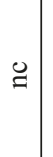 & $\stackrel{\Delta}{\sigma}$ & สิ & $\mid \begin{array}{r}\vec{v} \\
\vec{v}\end{array}$ & $\stackrel{\mathscr{g}}{\Xi}$ & $\stackrel{+}{\sim}$ & $\mathscr{\Xi}$ & $\mathscr{g}$ & $\stackrel{0}{\Xi}$ & $\vec{\lambda}$ \\
\hline 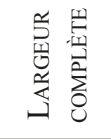 & $\hat{\vec{i}}$ & $\begin{array}{l}\infty \\
0 \\
0\end{array}$ & $\vec{n}$ & $\hat{6}$ & $\overrightarrow{0}$ & \begin{tabular}{|l|}
0 \\
$i$
\end{tabular} & $\stackrel{0}{\cong}$ & $\stackrel{\infty}{m}$ & $\stackrel{0}{0}$ & $\Rightarrow$ & $\begin{array}{l}\stackrel{+}{*} \\
\forall\end{array}$ & $\mathscr{g}$ & $\vec{n}$ & $\stackrel{\mathscr{g}}{\mathscr{G}}$ & ? & $\mathscr{g}$ & $\mid \begin{array}{l}n \\
0 \\
0\end{array}$ & $\stackrel{g}{g}$ & in & $\ddot{g}$ & $\mathscr{J}$ & $\mathscr{g}$ & $\stackrel{n}{*}$ \\
\hline 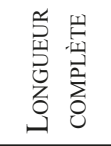 & $\vec{f}$ & $\mid \begin{array}{l}\infty \\
0 \\
0 \\
\tilde{e}\end{array}$ & సે & $\approx$ & $\begin{array}{c}n \\
m \\
m\end{array}$ & $\hat{\vec{\lambda}}$ & & $\begin{array}{l}\hat{\infty} \\
\tilde{m}^{0}\end{array}$ & $\frac{0}{m}$ & $\begin{array}{l}n \\
\tilde{e} \\
0\end{array}$ & $\left|\begin{array}{l}\infty \\
0 \\
0 \\
n\end{array}\right|$ & $\stackrel{\infty}{\sim}$ & $\frac{m}{m}$ & $\begin{array}{l}n \\
\text { ry }\end{array}$ & $m$ & m & $\tilde{m}$ & $\vec{\gamma}$ & q & $\mathscr{g}$ & $\mathscr{g}$ & $\mathscr{\Xi}$ & $\begin{array}{l}n \\
\text { f } \\
\text { fon }\end{array}$ \\
\hline 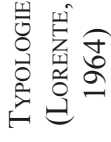 & - & $=$ & $\exists$ & $\exists$ & $\exists$ & $\exists$ & $\Xi$ & $\exists$ & $\exists$ & $=$ & $\exists$ & $\Xi$ & $\Xi$ & $\Xi$ & $\Xi$ & $\Xi$ & $\exists$ & $\exists$ & $\Xi$ & $\Xi$ & - & - & $\Xi$ \\
\hline 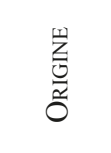 & 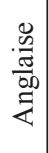 & 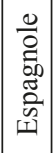 & 莞 & 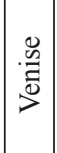 & 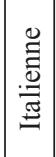 & 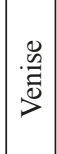 & 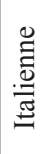 & 咅 & 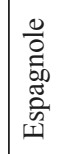 & $\mid$\begin{tabular}{c|}
0 \\
0 \\
0 \\
5 \\
$\tilde{0}$ \\
0 \\
0 \\
0
\end{tabular} & 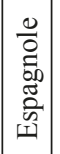 & 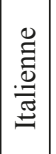 & 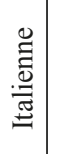 & 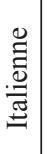 & 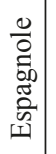 & 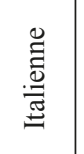 & 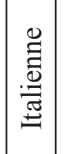 & $\begin{array}{c}0 \\
0 \\
0 \\
5 \\
\tilde{0} \\
0 \\
0 \\
19\end{array}$ & 离 & $\mid$\begin{tabular}{c|}
$\frac{0}{0}$ \\
0 \\
0 \\
$\tilde{0}$ \\
0 \\
0 \\
01
\end{tabular} & \begin{tabular}{|c|}
0 \\
0 \\
0 \\
0 \\
0 \\
$\tilde{0}$ \\
0 \\
0 \\
1
\end{tabular} & 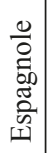 & 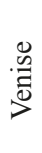 \\
\hline 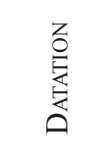 & $\begin{array}{l}0 \\
0 \\
o \\
+1 \\
\vdots \\
0 \\
0 \\
0\end{array}$ & 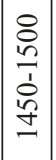 & $\stackrel{8}{8}$ & 号 & $\begin{array}{l}0 \\
n \\
n\end{array}$ & $\begin{array}{l}8 \\
: \\
-1\end{array}$ & 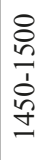 & $\begin{array}{l}8 \\
8 \\
n \\
0 \\
0 \\
n \\
n\end{array}$ & 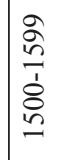 & 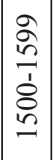 & $\mid \begin{array}{l}2 \\
0 \\
n \\
o \\
0 \\
n \\
-1\end{array}$ & 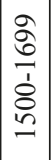 & 号 & 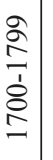 & 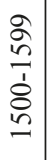 & 온 & : & $\begin{array}{l}2 \\
2 \\
\\
8 \\
0 \\
n\end{array}$ & $\begin{array}{l}8 \\
8 \\
0 \\
8 \\
8 \\
n \\
n\end{array}$ & 竎 & 辛 & 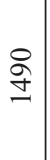 & $\stackrel{8}{\circ}$ \\
\hline 总 & $\begin{array}{l}0 \\
\infty \\
\infty \\
\infty \\
\vec{d}\end{array}$ & 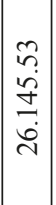 & 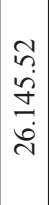 & $\mid \begin{array}{c}\vec{j} \\
\dot{q} \\
\dot{d}\end{array}$ & 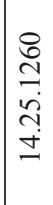 & $\begin{array}{l}n \\
\stackrel{n}{\dot{m}} \\
\dot{d} \\
\dot{d}\end{array}$ & $\begin{array}{l}\infty \\
\stackrel{m}{m} \\
\stackrel{m}{0} \\
\stackrel{0}{0}\end{array}$ & 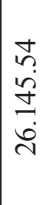 & 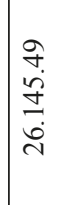 & 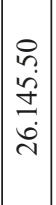 & 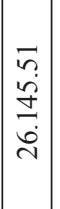 & $\mid \begin{array}{c}\tilde{D} \\
\stackrel{0}{m} \\
\dot{\sigma} \\
\dot{0}\end{array}$ & $\mid \begin{array}{c}\hat{m} \\
\dot{m} \\
\dot{0}\end{array}$ & $\begin{array}{l}\stackrel{0}{\rightarrow} \\
\underset{m}{j} \\
\dot{0}\end{array}$ & $\begin{array}{l}\vec{N} \\
\qquad \\
+ \\
\dot{f} \\
i\end{array}$ & $\begin{array}{l}\stackrel{f}{\sigma} \\
\stackrel{\sigma}{\sigma}\end{array}$ & $\begin{array}{l}\infty \\
\underset{\exists}{\Xi} \\
\end{array}$ & $\hbar$ & $\begin{array}{l}\infty \\
\stackrel{\infty}{=} \\
ت \\
ت \\
ت\end{array}$ & 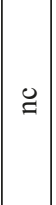 & $\mathscr{g}$ & 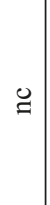 & 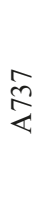 \\
\hline 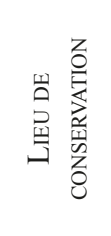 & $\underline{m}$ & $\stackrel{t}{\underline{m}}$ & $\stackrel{\underline{T}}{\Sigma}$ & $\overrightarrow{\mathrm{T}}$ & $\underline{5}$ & $\underline{\underline{\Sigma}}$ & $\underline{\underline{y}}$ & $\stackrel{\varphi}{\Sigma}$ & $\stackrel{-\varphi}{\Sigma}$ & $\underline{\underline{m}}$ & $\underline{T}$ & 䛼 & 嘼 & $\stackrel{-5}{\Sigma}$ & $\underline{m}$ & 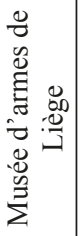 & 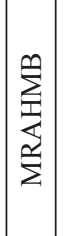 & 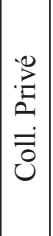 & 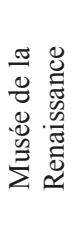 & 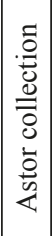 & 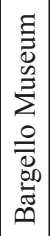 & 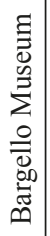 & 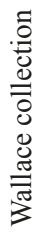 \\
\hline 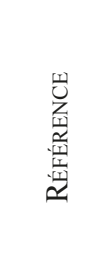 & $\begin{array}{l}\vec{b} \\
0 \\
0 \\
2 \\
\sigma \\
\overline{0} \\
0\end{array}$ & 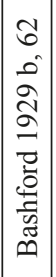 & 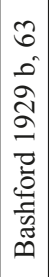 & 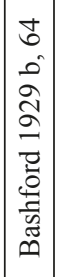 & 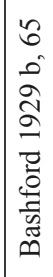 & 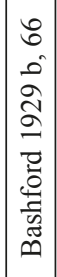 & 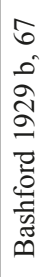 & 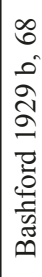 & 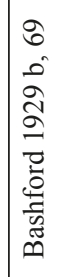 & 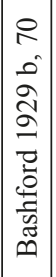 & 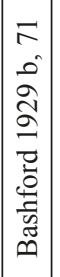 & 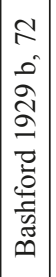 & 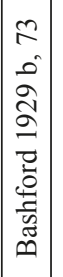 & 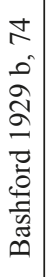 & 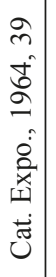 & 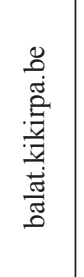 & 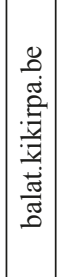 & 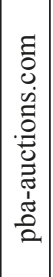 & 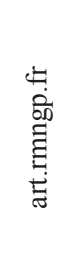 & 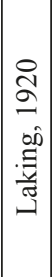 & 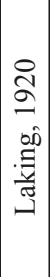 & 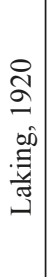 & 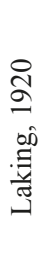 \\
\hline
\end{tabular}

Gladius, XXXIX (2019), pp. 109-126. ISSN: 0436-029X. https://doi.org/10.3989/gladius.2019.06 


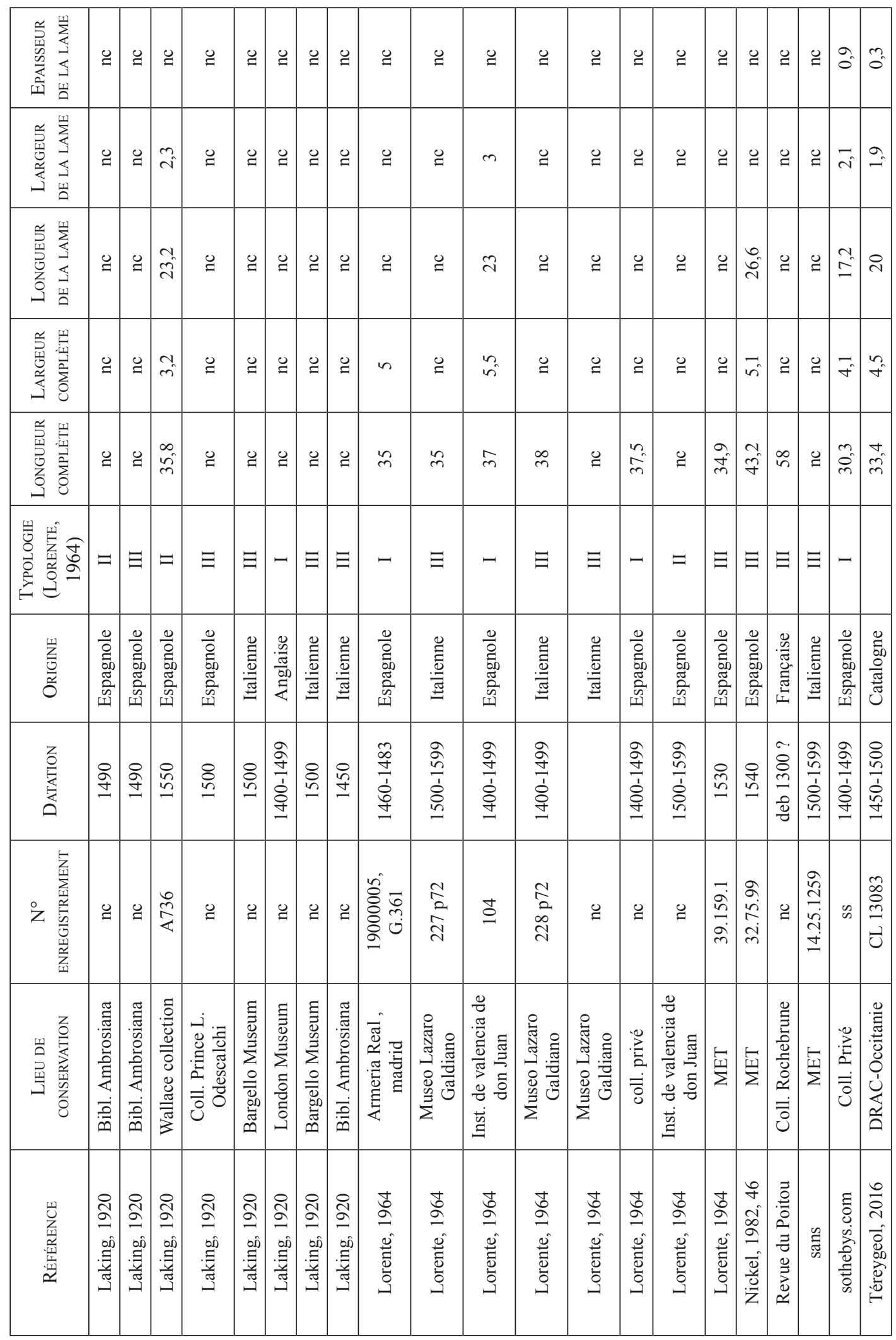




\begin{tabular}{|c|c|c|c|c|c|c|c|c|c|c|c|c|c|c|c|c|c|c|c|c|c|c|c|c|c|}
\hline $\begin{array}{l}n \\
\tilde{0} \\
\infty\end{array}$ & \begin{tabular}{|c|}
$\cdot \overrightarrow{\bar{\sigma}}$ \\
$\dot{\bar{\sigma}}$
\end{tabular} & & $\bar{z}$ & & & & & & & & & & & & & & & & & & & & & & \\
\hline$\ddot{0}$ & & 覀 & & & & & & & & & & & & & & $\stackrel{\circ}{\bar{z}}$ & & & & & & & & & \\
\hline 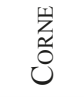 & & & & $\bar{z}$ & $\bar{z}$ & $\dot{\bar{\sigma}}$ & & $\bar{\Xi}$ & & & & & & & $\overline{\bar{\sigma}}$ & & $\bar{\Xi}$ & & & & & & 言 & & \\
\hline 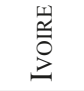 & & $\bar{z}$ & $\bar{z}$ & & & & & $\bar{\Xi}$ & & & & & & & & & & $\bar{z}$ & & $\overline{\bar{\sigma}}$ & & & & $\bar{\Xi}$ & $\bar{\sigma}$ \\
\hline oี & & $\overline{0}$ & $\bar{\sigma}$ & & $\overline{0}$ & & $\bar{\sigma}$ & $\ddot{\bar{\sigma}}$ & & & & & & $\dot{0}$ & & $\overline{0}$ & & '̄ & & $\bar{\Xi}$ & & & & $\bar{z}$ & $\overline{0}$ \\
\hline 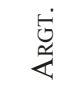 & & & & & & & & & & & & & $\Xi$ & & & & & $\bar{z}$ & & & & & & & \\
\hline 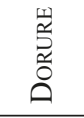 & & $\bar{\Xi}$ & & $\bar{z}$ & & & $\overline{0}$ & 言 & & & & & ' & & & & & & & & & & & & \\
\hline 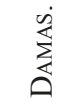 & & & & & & & $\overline{\bar{O}}$ & $\ddot{\Xi}$ & & & & & & $\bar{z}$ & & & & & & $\vec{z}$ & & & & & \\
\hline 画 & & & & & & & & & & & & & $\bar{\Xi}$ & & & & & & & & & & & & \\
\hline 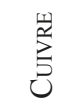 & $\bar{z}$ & & & & & & & & & & & & & & & & & & & & & & & & \\
\hline $\begin{array}{l}z \\
\\
\\
\\
\end{array}$ & & & & $\bar{z}$ & $\bar{\Xi}$ & 衣 & $\bar{\Xi}$ & '̄ & & & & & '̄ & & $\bar{\Xi}$ & & $\bar{\Xi}$ & $\bar{\Xi}$ & & & & & ż & & \\
\hline 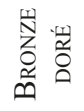 & & & & & & & $\exists$ & & & & & & $\cdot \bar{z}$ & $\bar{\Xi}$ & & & & & & & & & & & \\
\hline 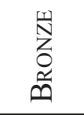 & & $\bar{z}$ & & & & & $\bar{\Xi}$ & 言 & & & & & $\bar{z}$ & $\overline{\bar{\sigma}}$ & & & & & & & & & $\bar{z}$ & & \\
\hline 鲎㟧 & & $\overline{0}$ & & $\overline{0}$ & $\overline{0}$ & 言 & 言 & : & & & $\overline{0}$ & & $\overline{0}$ & & & $\bar{z}$ & & $\bar{z}$ & $\bar{\Xi}$ & $\ddot{\bar{\sigma}}$ & $\bar{z}$ & $\bar{\sigma}$ & & & \\
\hline 舀芫 & & & $\bar{z}$ & & & & & & & & & & $\bar{z}$ & & & $\bar{\Xi}$ & & $\bar{z}$ & $\bar{z}$ & $\overrightarrow{\bar{\sigma}}$ & $\bar{z}$ & $\bar{\sigma}$ & & $\overline{0}$ & 'z \\
\hline 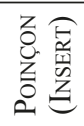 & $\tilde{z}$ & z̃ & z̃ & zี & z̃ & ฉี & z̃ & ฉ̃ & そ̃ & z̊ & हี & $\overline{\bar{\partial}}$ & zี & 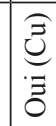 & z̃ & z̃ & 艺 & $\begin{array}{l}\tilde{0} \\
z \\
z\end{array}$ & 芩 & z̃ & $\dot{\bar{z}}$ & $\bar{z}$ & z̃ & z̃ & ż \\
\hline $\begin{array}{l}0 \\
0 \\
0 \\
0 \\
0 \\
0\end{array}$ & 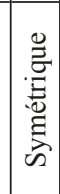 & 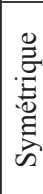 & 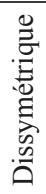 & 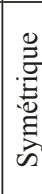 & 兽 & 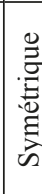 & 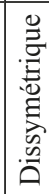 & 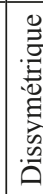 & 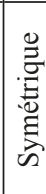 & 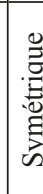 & 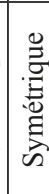 & 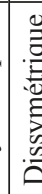 & 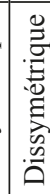 & 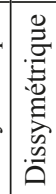 & $\mathscr{g}$ & 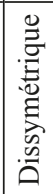 & s & 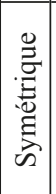 & 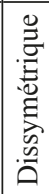 & 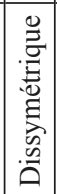 & 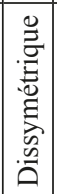 & 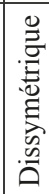 & 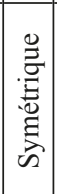 & 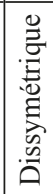 & 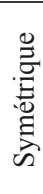 \\
\hline 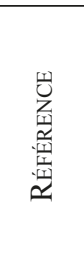 & 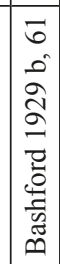 & 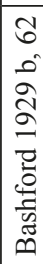 & 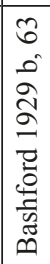 & 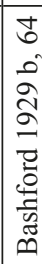 & 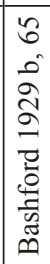 & 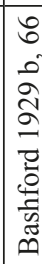 & 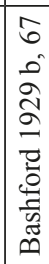 & 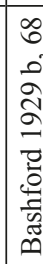 & 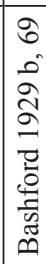 & 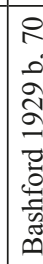 & 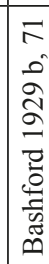 & 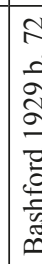 & 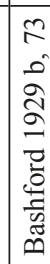 & 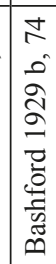 & 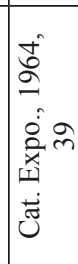 & 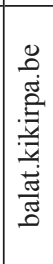 & 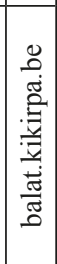 & 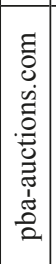 & 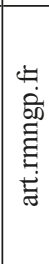 & 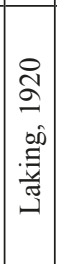 & 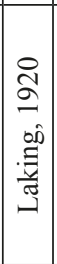 & 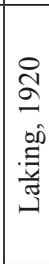 & 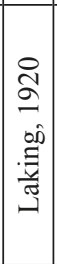 & 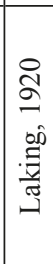 & 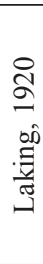 \\
\hline
\end{tabular}




\begin{tabular}{|c|c|c|c|c|c|c|c|c|c|c|c|c|c|c|c|c|c|c|c|}
\hline $\begin{array}{ll}\frac{n}{n} \\
\stackrel{n}{n}\end{array}$ & & & & & & & $\overline{5}$ & & & & & & & & & & & & '̄ \\
\hline 0 & & & & & & & & & & & & & & & $\bar{z}$ & & & & \\
\hline 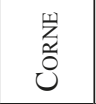 & & & & & & & & & & & & & & & & & & $\bar{z}$ & \\
\hline 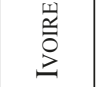 & & & $\bar{z}$ & & & & $\overline{\bar{\sigma}}$ & & $\overline{5}$ & & 5 & $\bar{\xi}$ & & $\Xi$ & $\bar{\Xi}$ & ठ̃ & $\bar{\Xi}$ & & \\
\hline ő & & & & & & & $\overline{\bar{\sigma}}$ & & $\bar{\xi}$ & & & $\bar{\xi}$ & & "E & "̄ & $\bar{z}$ & & & \\
\hline $\begin{array}{l}\dot{0} \\
\stackrel{\tilde{x}}{4}\end{array}$ & & $\bar{\sigma}$ & & $\overline{3}$ & & & & & & & & & & & & $\bar{z}$ & & & \\
\hline 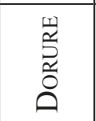 & & & & & & & & & & & & & & & & $\tilde{\sigma}$ & & & $\bar{z}$ \\
\hline 离 & & & & & & & & & & & & & & & $\bar{z}$ & & & $\bar{z}$ & \\
\hline 苞 & & $\bar{z}$ & & & & & & & & & & & & & & & & & \\
\hline 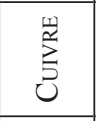 & & & & & & & & & & & & & & z & & $\bar{z}$ & & & \\
\hline 总 & & & $\bar{z}$ & & & & $\bar{\sigma}$ & $\bar{\Xi}$ & & & & & & $\bar{z}$ & & & & & 'z \\
\hline 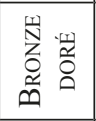 & & & & & & & & & & 5 & & & & & & & & & \\
\hline $\begin{array}{l}\text { 㟧 } \\
\text { 邑 }\end{array}$ & & & & '̄ & & & & & & 5 & & & & & & & & & \\
\hline 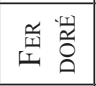 & & & & & & & $\bar{\vdots}$ & ठे & & & 5 & $\bar{\Xi}$ & & & $\bar{z}$ & & & $\overline{0}$ & \\
\hline 畄嶧 & $\bar{z}$ & $\overline{0}$ & & & & & $\bar{z}$ & $\overline{\bar{o}}$ & E & 5 & & & 言 & & $\bar{z}$ & & & $\bar{z}$ & \\
\hline 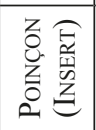 & 穵 & 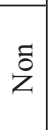 & õ & & $\bar{a}$ & & $\tilde{\sigma}$ & 客 & E & & & $z$ & שِ & $\frac{\tilde{z}}{\bar{z}}$ & ฐี & ฉ̊ & 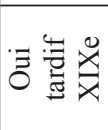 & $\bar{z}$ & 兽 \\
\hline 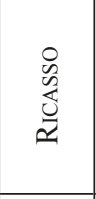 & 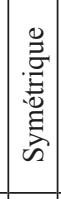 & 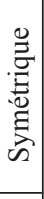 & 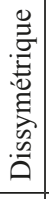 & $\infty$ & 吾 & 总 & 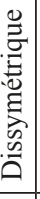 & 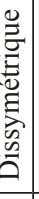 & 足 & | & & $\oiiint$ & 吾 & 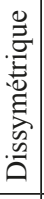 & 童 & 胥 & $\mathscr{g}$ & & 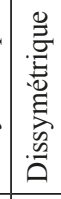 \\
\hline 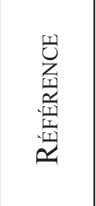 & 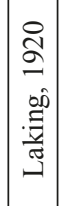 & & $\delta$ & $\tilde{\sigma}_{00}$ & & $\frac{0,0}{\underline{z}}$ & & 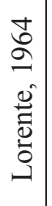 & & & & 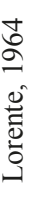 & & & 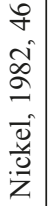 & $\begin{array}{l}3 \\
\bar{z} \\
0\end{array}$ & 嵒 & & 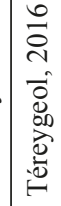 \\
\hline
\end{tabular}

Gladius, XXXIX (2019), pp. 109-126. ISSN: 0436-029X. https://doi.org/10.3989/gladius.2019.06 


\section{CONCLUSIONS}

À l'échelle du site où la dague a été trouvée, nous pourrions nous perdre en conjectures sur la présence de cet objet en mine : un vol, une perte, un dépôt propitiatoire ? En l'état actuel des connaissances, le plus simple est de supposer la présence de gardes assurant le contrôle de l'exploitation. L'existence du castrum renforce cette hypothèse. Son contexte de découverte et la datation intrinsèque de l'objet fournissent un terminus post quem pour l'activité minière située entre la fin du XV $\mathrm{V}^{\mathrm{e}}$ siècle et le début du XVI ${ }^{\mathrm{e}}$ siècle qui s'accorde avec les autres éléments connus. La date de fabrication, si l'on s'en tient aux indications fournies par les restes du fourreau, prend place dans la seconde moitié du $\mathrm{XV}^{\mathrm{e}}$ siècle. Ce point reste cependant à éclaircir.

$\mathrm{Au}$ regard de la typologie des dagues à oreilles, celle de Castel-Minier se place comme un objet unique à plusieurs titres. Elle est d'abord la seule à être découverte dans un contexte archéologique connu. Ensuite, elle est accompagnée de son fourreau, élément particulièrement rare dans les collections. Enfin, jusqu'à présent, seuls étaient connus des modèles à destination d'une élite, et les auteurs s'accordaient pour voir dans ce type de dague une arme de prestige (Blair, 1970). Même si elle reste un objet de belle facture, notre dague montre également la perméabilité des effets de mode dans la société. Sans grande surprise, elle ne répond pas aux standards définis pour ce type d'objet. Mieux, il semble possible de définir avec elle, et en regard des représentations picturales, un quatrième type d'origine catalane. Ce dernier avait été proposé par Bashford (Bashford, 1929a: 52; 1929b: 66) en son temps, mais non repris dans la typologie de Rodríguez Lorente, car il n'existait pas de témoignage matériel du modèle. C'est maintenant chose acquise.

\section{REMERCIEMENTS}

La fouille archéologique bénéficie du soutien de la Direction Régionale des Affaires Culturelles d'Occitanie (France), de la commune d'Aulus-les-Bains (France) et de la Wilhelm-Mommertz-Stiftung (Allemagne). La restauration de la dague a été assurée à Toulouse par Materia Viva que nous remercions. Les radiographies ont été faites gracieusement par Bernard Rattoni du service de contrôle radiographique du CEA à Saclay. L'identification du bois est l'œuvre de Marie-Claude Saad travaillant au Laboratoire Archéologie et science de l'antiquité (UMR 7041 ArScan, CNRS) à l'université Paris-Nanterre qui a en charge les volets palynologique, anthracologique et xylogogique de la fouille de Castel-Minier. Nous lui adressons nos sincères remerciements pour nous avoir communiqué par avance ses résultats. Merci enfin au musée national de la Renaissance et au musée royal de l'armée et d'histoire militaire de Bruxelles pour nous avoir fourni les informations sur les dagues à oreilles de leur collection respective.

\section{BIBLIOGRAFÍA}

Arminjon, C. (1998): Métal, vocabulaire technique. Paris, Éditions du Patrimoine.

Bashford, D. (1929a): The Collection of European Daggers of Jean Jacques Reubell. New York, The Metropolitan Museum of Art.

Bashford, D. (1929b): Catalogue of European Court Swords and Hunting Swords: Including the Ellis, De Dino, Riggs, and Reubell Collections. New York, The Metropolitan Museum of Art.

Blair, Cl. (1970): «A royal swordsmith and damascener: Diego de Caias». The Metropolitan Museum Journal, 3: 149-198. 
Camón Aznar, J. (1951): Guía del Museo Lázaro Galdiano. Madrid, Fundación Lázaro Galdiano.

Charbonneau-Lassay, L. (1918): «Dague et épée dites "stradiotes »». Revue du Bas-Poitou, 81: 297302.

Cosson, Ch.-A. De (1901): Le cabinet d'armes de Maurice Talleyrand-Périgord, duc de Dino. Paris.

Cowgill, J.; De Neegaard, M. and Griffiths, N. (1987): Knives and scabbards, medieval finds from excavations in London, vol. 1. Londres, Boydell press.

Dandridge, P. and Wypyski, M. T. (2011): «Sword and dagger associated with the crusades Part II: a technical study». The Metropolitan Museum Journal, 46: 145-153.

Espinosa Martin, C. (2015): Colección Lázaro de Nueva York, Catálogo de la Exposición. Madrid, Museo Lázaro Galdiano.

Florit y Ariscun, J. M. y Sanchez Canton, F. J. (1927): Catálogo de las armas del Instituto de Valencia de don Juan. Madrid, Instituto de Valencia de don Juan.

Grancsay, S. (1940): «A damascened eared dagger by Diego de Caias». The Metropolitan Museum of Art bulletin, 35 (8): 160-161.

Laking, G. F. (1920): A record of European armour and arms, vol. III. Londres, Bell and son Ltd.

Malus, J. (1600): Recherche et Descouverte des mines des montagnes Pyrénées. Toulouse, Bourneton.

Mugueta, I. (2004): «Política monetaria en Navarra bajo el reinado de los primeros Evreux (13281349)». En la España Medieval, 27: 77-104.

Nickel, H.; Pyhrr S. W. and Tarassuk L. (1982): The art of chivalry, European arms and armor from the metropolitan museum of art. New York, The Metropolitan Museum of Art.

Oakeshott, E. (1960): The Archaeology of Weapons: Arms and Armour from Prehistory to the Age of Chivalry. Woodbridge, The Boydell Press.

Oakeshott, E. (1980): European weapons and armour, from Renaissance to the industrial revolution. Woodbridge, The Boydell Press.

Rodríguez Lorente, J. J. (1964): «The $\mathrm{XV}^{\text {th }}$ century ear dagger. Its hispano-moresque origin». Gladius, III: 67-87.

Téreygeol, F. (2016): Le Castel-Minier (Aulus-les-Bains, 09), rapport de fouille programmée. Toulouse, DRAC-SRA Midi-Pyrénées.

Verna, C. (1996): «Une nouvelle page de l'histoire des mines d'argent européennes : le cas des Pyrénées centrales (XIVe - XVe siècle)». Bulletin annuel de la Société Ariégeoise Sciences Lettres et Arts: 201-232.

Recibido: 28-02-2019

Aceptado: 11-07-2019 